\title{
HOW TO REINTERPRET AN ALPINE ECO-MONSTER. APPLICATION OF THE METHOD OF CHOICE EXPERIMENTS FOR THE DESIGN OF A REUSE PROJECT
}

\author{
MARTA BOTTERO ${ }^{1}$, ANTONIO DE ROSSI $^{2}$, ANDREA PONZETTO $^{2} \&$ DAVIDE VIANO $^{2}$ \\ ${ }^{1}$ Politecnico di Torino, Department of Regional and Urban Studies and Planning. \\ ${ }^{2}$ Politecnico di Torino, Department of Architecture and Design.
}

\begin{abstract}
This paper deals with the role of evaluation methods for supporting the design process of sustainable urban and territorial transformations. In particular, the article considers the technique of Choice Experiments (CE) and it proposes a real application of the method for driving the decision-making process related to the reuse project of an abandoned building located in the Italian Alps. The evaluation model is based on different attributes of the reuse project, both tangible and intangible, including internal organization, external areas, accommodation structures and cost. In the evaluation, a questionnaire has been developed for the investigation of the preferences of potential users with reference to alternative reuse scenarios. The results of the application allowed us to determine the importance of the different attributes for the definition of the reuse strategy as well as the economic value of the selected strategy. The study proposed in this paper represents an innovative context of application of the CE method, regarding the economic evaluation of architectural buildings and landscape. A second innovative element of the present research concerns the use of the Choice Experiments approach for supporting the design of alternative solutions for a complex decision making problem.

Keywords: Decision making, Strategic design, Adaptive reuse, Stated preferences, Regeneration processes, Mountain.
\end{abstract}

\section{INTRODUCTION}

It is well known that sustainability assessment in the context of projects and plans is a complex task which is defined by a plurality of actors with different values and objectives, and characterized by a great deal of uncertainty. Taking into consideration this complexity, it is of particular importance to provide the decision makers, planners and designers with integrated evaluation tools, able to consider the multiplicity of visions when dealing with urban and territorial transformations, to include the opinions and the needs of the different stakeholders involved and to assess the impacts and the consequences of each decisions [1], [2]. In particular, starting from the aforementioned complexity, the present paper aims at investigating the role of evaluation methods in supporting the definition of project strategies, with specific attention to the design of alternative solutions and to answer the question 'will it work?' for the different proposals. In the analysis, particular attention will be devoted to a careful framing of the problem and to a definition of the objectives one wants to solve in order to understand if minimum objectives will be achievable. Among the evaluation methods for assessing sustainability in the context of projects/plans/programmes, a very important role is played by the method of the Choice Experiments (CE). CE method is a procedure designed to generate survey protocol in order to reveal the factors that influence individual preferences. The first applications of the method were related to the field of transport and marketing economics [3]. Later on, the method has been adapted for environmental and landscape evaluation purposes [4]. A central feature of this approach is that the utility derived from a good or service can be decomposed into 'part-worths' relating to different attributes of that good or service [3]. This ability to investigate the 'part-worths' of a good is well suited to the nature of landscape and 
architectural heritage. Conducting a CE will allow to understand which are the most important components of the goods or services under investigation according to the people preferences and values and which is the trade-offs they are prepared to make among the different aspects describing the goods/services [5]. Moreover, a key element of the CE approach consists in asking individuals about their preferences over different hypothetical alternative scenarios. This is of particular importance in the context of landscape and architectural heritage where people's perception and values are crucial in the decision-making processes related to the conservation, valorisation and management of asset [6]. More generally, CE can offer a valid contribution in sustainability assessment in a broad context as they allow to include not only multidisciplinary aspects (environmental, social and economic dimensions) but also cultural and value-based elements [23]. Indeed, CE can provide useful support to Decision Makers who have to take decisions for making more sustainable societies.

This article investigates in detail the use of the CE method for supporting a real-world decision making process. In particular, in this paper, we analyze the development of the ski resort in Saint Gréé di Viola, a small town in Northern Itay, with specific attention to the project named 'La Porta della Neve' (The Snow Door), a huge architecture constructed in the 1970s with a multi-functional destination that now is abandoned. Today this building can be defined an 'eco-monster'. This term refers to a neologism often used in the national and international debate for denoting buildings or complexes of buildings grossly incompatible with the natural environment and with the surrounding landscape. In this sense, it seems to be of particular importance to find innovative solutions able to guarantee sustainability for this kind of situations which are based on the consideration of the full range of impacts at stake. In specific, in order to face the problem of this site and to propose a new life for the area, the work focuses on a preliminary feasibility analysis for the reuse project of this building [7].

\section{METHODOLOGICAL BACKGROUND}

The choice of intervention in the context of urban and territorial transformations requires the development of a multidisciplinary assessment to verify if the project is suitable for meeting the initial objectives. This assessment is generally called 'feasibility study' and is organized according to three main sections: (i) analysis of the technical feasibility and urban planning; (ii) analysis of the economic and financial feasibility; (iii) analysis of management feasibility.

Within the context of the financial and economic feasibility, a very important role is covered by the demand and supply analysis. The demand analysis is preliminary and essential for any economic initiative and it allows us to understand the conditions of the area in which the operation will be located, specifying the market potential of the project. Conversely, with the supply analysis it is possible to have a picture of the competitive scene in which the operation will take place. It is also useful to gather information about the performances of possible competitors by means of benchmarking analysis.

Another important issue in this context is the construction of a model of socio-economic analysis which allows the public interest to be pointed out. This paper investigates the role that $\mathrm{CE}$ can cover in such economic models.

In particular, the CE method is a technique of statistical multivariate analysis and it aims to study individual choices using preferences expressed about various profiles, i.e. several versions of a product or service [3]. As stated by Louviere et al. [8], CE are based on a welltested theory of choice behaviour called Random Utility Theory (RUT, [9]) that is able to offer an explanation of the choice behaviour of human beings. 
The method uses a decompositive approach to establish the importance of different elements (attributes) in the determination of the preference of a good or service by the course of an individual. From a methodological point of view, the development of the technique includes different steps that are described as follows.

Step 1: Definition of a set of attributes or features describing the good, service, project or policy, each taking a number of pre-specified levels.

Step 2: Combination of these levels and attributes to build up descriptions of hypothetical bundles, using experimental design techniques.

Step 3: Development of a questionnaire for asking individuals to state their preferences over these alternatives, using a number of different protocols.

Step 4: Analysis of the individual responses and prioritization among the different combinations of features.

Among the main strengths of the $\mathrm{CE}$, it is possible to recall the possibility of providing a comprehensive conceptualization of the entire system under investigation, the capacity of representing real-world decision making processes, avoiding unfeasible or unrealistic options, and the faculty of considering in a flexible way different protocols of eliciting preferences. For the aforementioned reasons, CE are able to provide a deep understanding of how people make their choices and different applications of the method exist, not only in marketing, but also in other fields of applied economics. It has to be noticed that CE have been mostly used to estimate the value of environmental goods (e.g. [5], [4], [10]). Few applications exist dealing with the assessment of the value of landscape and cultural [6], [11], [12].

\section{CASE STUDY}

\subsection{Description of the site}

During the 1950s and 1960s of the XX century Italian people started to feel the pleasure of spending their holidays at the seaside or in the mountains. At that moment, it became natural for many families to consider the purchase of a second home for their vacations. The touristic experience had a standardization process: attractions, natural beauty, artistic and cultural heritage became part of a 'package' that modern tourists can use during their free time. At the same time, a lot of structures started to be built with the aim of supporting the tourism expansion and the alpine landscape changed because of the development of the winter sports [13], [14].

The case study under consideration in the present research is related to the building named 'La Porta della Neve' which is located in the site of Sain Grée di Viola in the Piedmon Region (Italy).

The tourist site was designed by the Italian engineer Giacomo Fedriani in 1964 with the aim of creating a new skiing resort. The area was initially composed by 6 buildings with 40 apartments each. In the early 1970s all the buildings were completed and in the mid-1970s a huge architecture was added to the system. It consisted in a large multi-functional complex, called 'La Porta della Neve'. It was a gigantic building with two main entrances: the first one was placed at the municipal road level, the other one was placed few meters higher, directly at the ski slopes level. The structure enclosed one hotel and different services, such as a cinema/theater, a supermarket, a gym, a bar, one indoor swimming pool, several boutiques and a restaurant dedicated to skiers, directly accessible from the ski slopes. 
During the late 1970s and early 1980s this building was the protagonist of the development of the overall site and other residential and retails structures were constructed. Unfortunately, in 1985 Fedriani decided to sell the whole complex because of personal problems. In 1989 the resort was placed in liquidation due to mismanagement. After the closure of the structure, a gradual process of abandonment of the whole locality began, also because of the lack of services and a very poor level of snow in the winter season induced by the climate change of the region. Today the entire country St. Gréé di Viola looks like a 'ghost town', made up of uninhabited condominiums and architectures in a state of decay (Fig. 1). In this context the former multi-functional complex constitutes a sort of eco-monster for which sustainable solutions should be formulated for re-launching the building and the town.

With the aim of revitalizing the site, a reuse project has been studied. The main idea of the project consists in the incorporation of new functions in the building in order to make 'La Porta della Neve' a large center totally focused on sports.

\subsection{Preliminary analysis}

Before the CE application, a series of analyses on the territory aimed at obtaining data and information on users and potential tourists intervention were carried out. This part of the evaluation is finalized at verifying the opportunity of considering the operation. This is a very important step of the overall decision-making process as it allows us to substantiate the project proposal. This phase can be developed according to three main steps: analysis of the demand, analysis of the supply and benchmarking.

\subsubsection{Analysis of the demand}

This analysis is based on the estimate of the total demand forecasting for the project proposal in order to understand the needs that must be solved with the operation in the specific market under consideration [15].

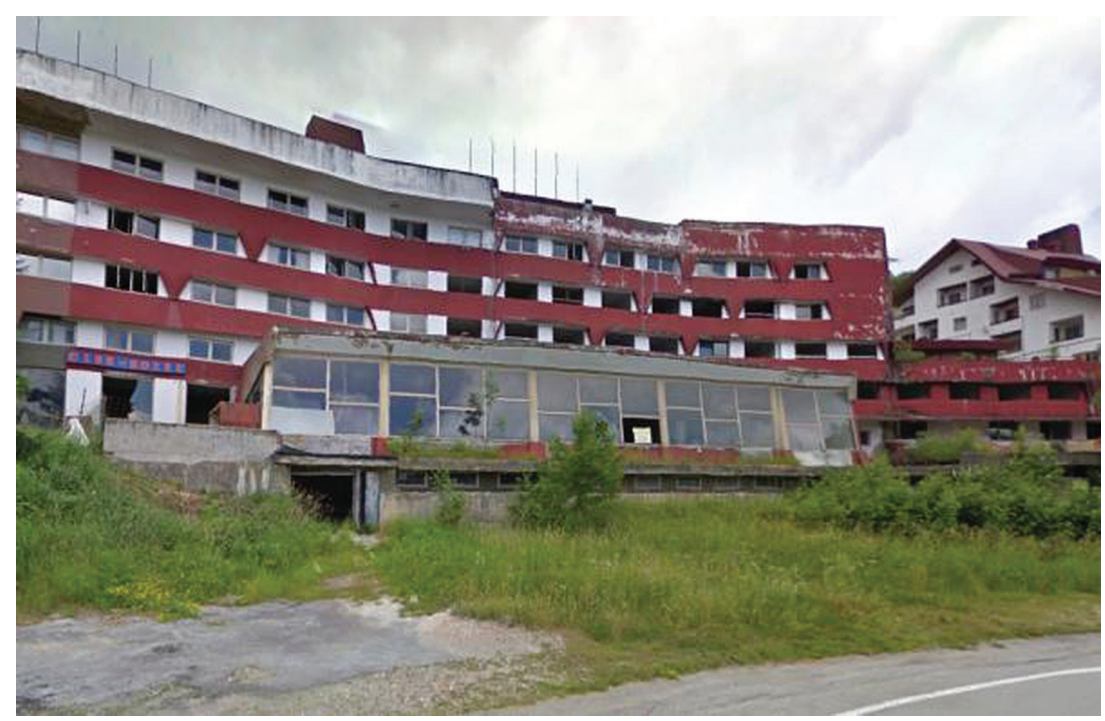

Figure 1: The building 'La Porta della Neve' in Saint Gréé di Viola. 
Firstly, the catchment area has been analyzed. In particular a map showing the isochronous curves of the different travelling times (every 30 minutes) required to reach Saint Gréé Viola from different cities has been created (Fig. 2).

We assumed that the catchment area was constituted by the sites from which Saint Gré di Viola is accessible in 210 minutes by car. In this case the catchment area considers 12 Provinces that are located in different Regions. Secondly, the analysis considered the trend of tourists arrivals in the area under investigation using the data provided by the Regional tourist office. According to this analysis, the area of Cuneo, where the project is located, is the third most important site in the Piedmont Region from the point of view of the tourists presences, after the city of Torino and the Lake Maggiore area. Moreover, a recent study developed by the Chamber of Commerce of the Province of Cuneo provides relevant information about the typology and the habits of the tourists in the area under examination. According to this study it is possible to state that the mountain resorts are placed at the first place from the point of view of the tourists presences of the Province, the tourist type who chooses the mountain is a man aged between 41 and 50 years or younger, the vacation lasts an average of 1-2 weeks, for overnight stay, foreigners especially prefer hotels whereas Italians normally own a second house in the area; finally tourists spend their time mostly practicing sports.

\subsubsection{Analysis of the supply}

After the analysis of the demand, the second element in the market research is the supply. In this context, it is of particular importance to develop an estimation of the potential competitors in the submarket under consideration. This will allow us to estimate the part of the total demand of the specific sub-market that will target the operation in exam. The analysis of the

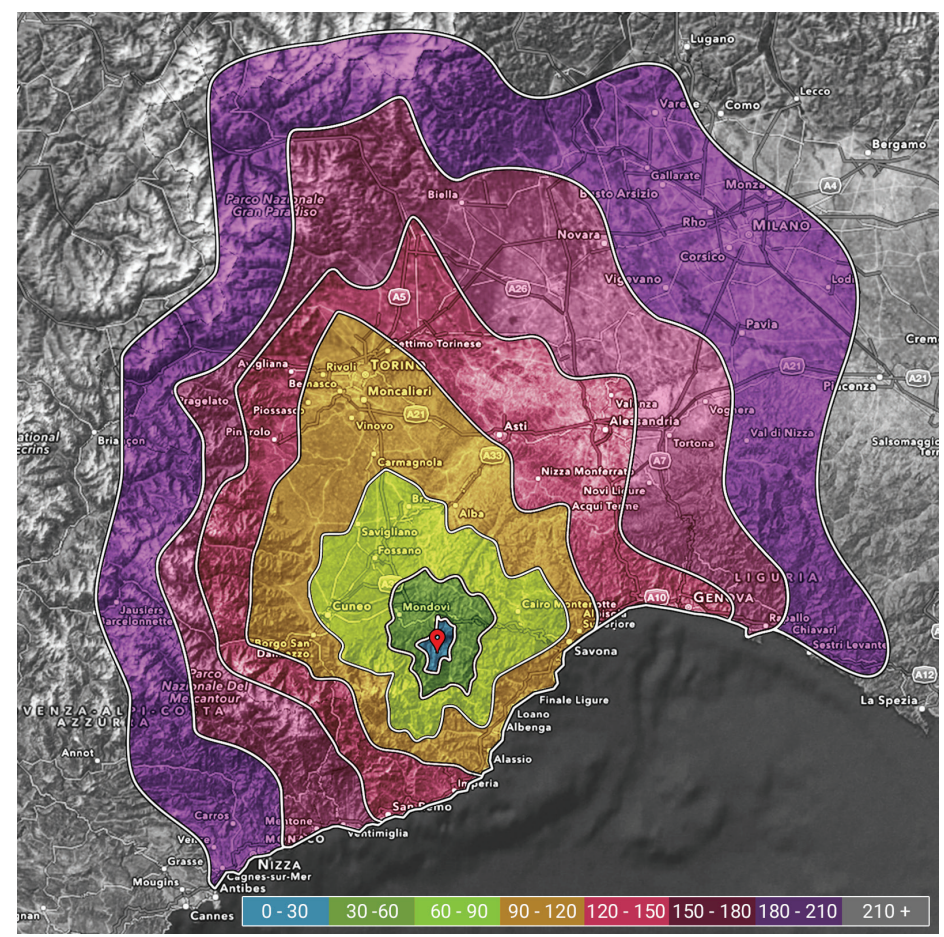

Figure 2: Map with isochronous curves in 30 minutes intervals from Saint Gréé di Viola. 
supply has been developed on the surrounding area by reducing the range to $60 \mathrm{~km}$ from Saint Gréé di Viola. In this area, the various sites that could be in competition with the operation under investigation have been taken into consideration. These sites are represented in the map of Fig. 3 and they have been arranged graphically according to different categories of sports and activities, namely climbing, skate park, bobsleigh, paintball, bike parks, funpark, SPA / wellness center. As is possible to see from Fig. 3, the climbing and SPA/Wellness center sites are quite diffused on the territory, especially in the Province of Cuneo, while the number of skateparks, bikeparks and paintball centers is more limited.

\subsubsection{Benchmarking}

The benchmarking was originated as tool for the analysis and comparison of different companies, in order to confront the performances of the enterprises with external realities. The main objective of the benchmarking exercise is to highlight the benefits and to understand the operational practices that led to the achievement of these performances. Over the past 20 years numerous attempts to apply this methodology to the domain of territory, landscape and architecture have been developed.

In the context of the present research, the benchmarking analysis consisted in the development of a survey aiming at identifying a series of similar goods to be compared to the project. In the present study, nine case studies have been chosen; these case studies are constituted by multi-purpose buildings with sports function located in Europe and constructed after the year 2000. More specifically, the benchmarking survey was divided into two phases. Firstly, the nine case studies have been investigated in order to recognize the guidelines according to which each project was developed. Secondly, these structures have been compared with the

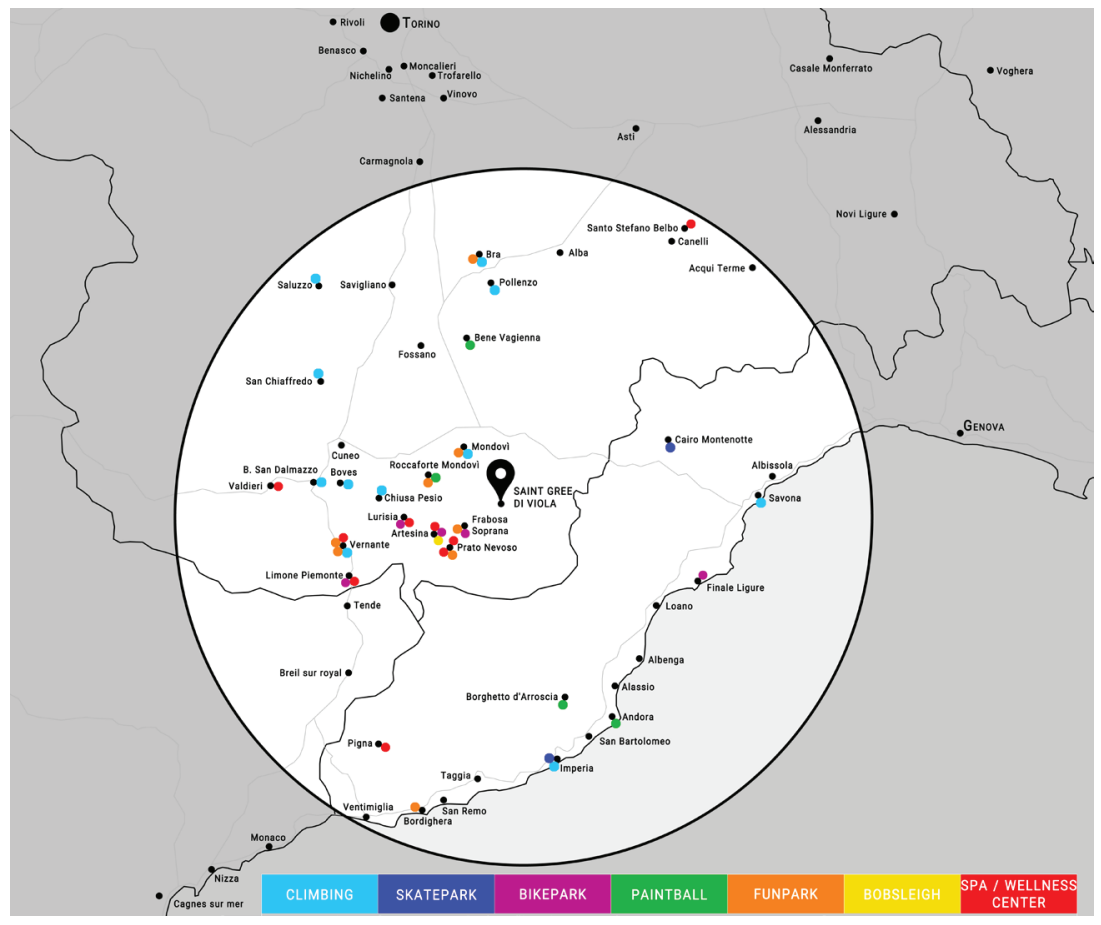

Figure 3: The map of the supply on the surrounding area to Saint Gréé di Viola. 
Table 1: Examination of the sport activities in the 9 case studies considered for the benchmarking analysis.

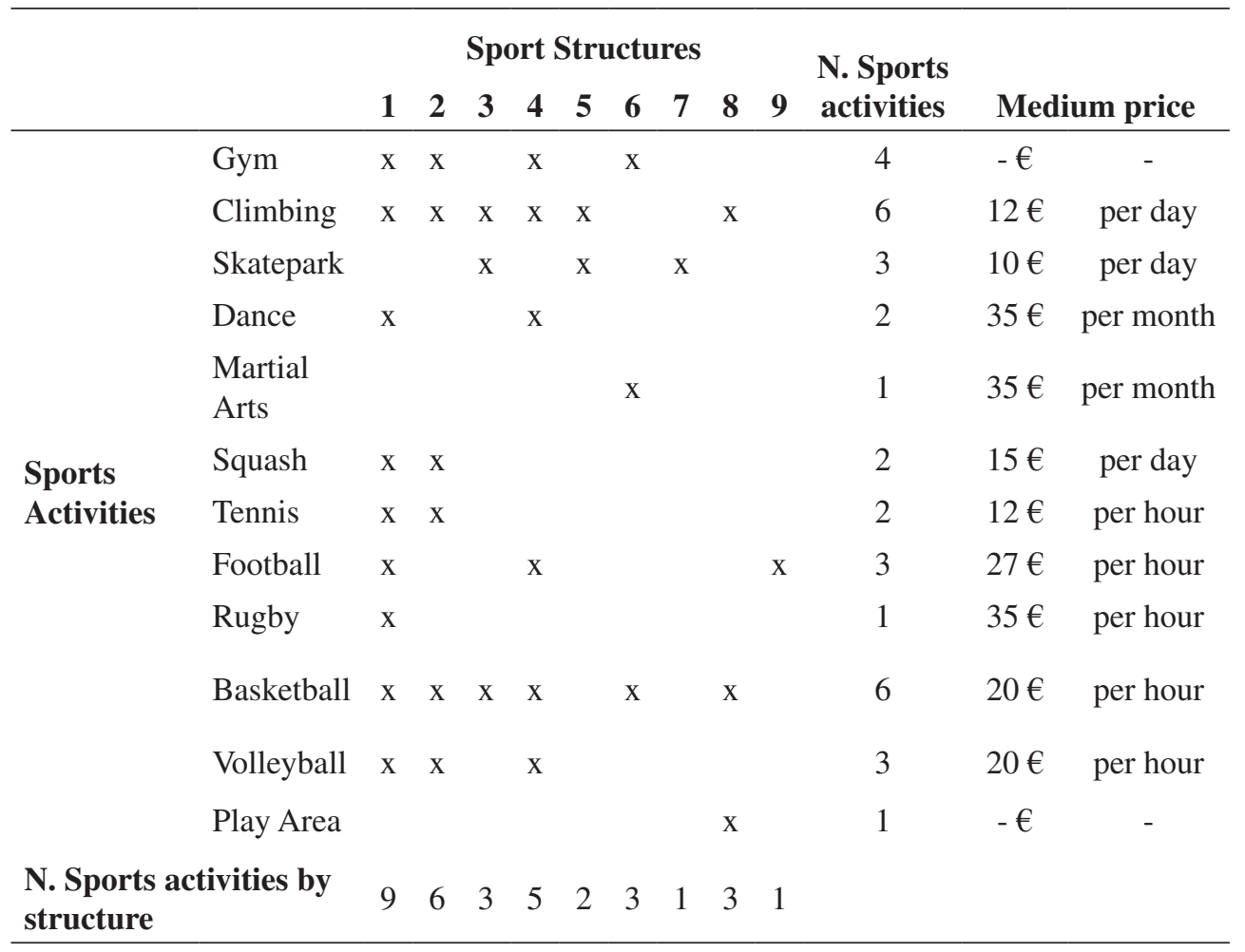

(1) Sport Center Jules Ladoumegue, Paris France; (2) Sport Center Dordrecht, Dordrecht Netherlands; (3) StreetDome, Haderslev Denmark; (4) Sport Center ZAC del Lilas Multi-Purpose, Paris France; (5) Mèrida Youth Factory, Mèrida Spain; (6) Sport Centre Pajol, Paris France; (7) ZAP'ADOS, Calais France; (8) Sport and Leisure Center, Saint Cloud France; (9) Sport Facility, Coimbra Portugal.

aim of understanding the number and the type of activities present and the related prices. Table 1 details the data and the results emerging from the benchmarking survey.

\section{APPLICATIONS OF CE TECHNIQUE}

\subsection{Experimental design}

As illustrated in Section 2, the first methodological step of the CE method is to define the attributes and levels. In this phase, it is necessary to select the characteristics of the objects or services under consideration (attributes) and to define the different entities (levels) that they can take. The combination of attributes and levels generates the profiles of choice that will be presented to respondents. The considered elements within the attributes and levels must be easy to understand by users and should remain limited in number.

In this case, six attributes have been identified, namely (i) accommodation, (ii) food, (iii) outdoor sports, (iv) staff, (v) indoor sports and (vi) cost . Each attribute has been associated to three levels that represent possible options for heritage management. 
The attribute 'accommodation' refers to the typology of lodging structures that can be included in the reuse project and it varies among three stars hotel, two star hotels and hostels.

The attribute 'food' concerns the kind of restaurant that could exist in the renovated building. In this case three levels were considered, namely restaurant and bar, bar and fast food and only fast food.

The attribute 'outdoor sports' considers the extension of the surface for outdoor activities in the external areas surrounding the buildings. This attribute varies among the levels high, medium and low.

The attribute 'staff' concerns the presence of specialized people following individuals when doing sport activities. This attribute varies among the levels high, medium and low.

The attribute 'indoor sports' regards the organization of the internal surfaces of the building for the development of the indoor activities. Three situations are envisaged, namely few possibilities of different sports in a large area, many possibilities of different sports in a limited space and an intermediate solution.

Finally. the attribute 'cost' considers the global expenditure for passing a week end in the site.

It shall be noted that the aforementioned attributes and levels consider the specific characters and values for the reuse of the project under investigation. Moreover, the structuring of the evaluation model has been designed with the help of a specific focus group where the proposed attributes and levels were discussed by several experts in the field of alpine architecture, landscape planning and economics and management in order to reach a common vision.

It has also to be noticed that the levels of the cost attribute were defined via a pre-test. In this pre-test a small sample of potential users was asked about the Willingness To Pay (WTP) for an hypothetical project scenario where the attributes were set at the maximum level. More in details, the respondents were asked to express their WTP for spending an 'all inclusive' weekend in the structure redesigned. The values thus obtained were further elaborated in order to produce the three levels considered in the final model.

In details, the attributes considered for the evaluation are defined in Table 2.

Given the number of attributes and levels (Table 1), there would have been too many possibilities (i.e. $3^{6}=279$ combinations) to use all them in the survey. In order to identify a minimum efficient set of combinations, an orthogonal fractional experimental design was followed [16].

This approach allows to select profiles that do not overlap, while ensuring data consistency for conducting the statistical analysis. The generation of the orthogonal design was made using the SPSS software (www.spss.com), where 18 combinations of attributes were selected.

\subsection{Questionnaire}

The subsequent step of the model consists in assessing the trade-offs across the project attributes identified in the first phase and their marginal value through eliciting people's preferences for the reuse characteristics.

In this case, the preferences have been collected using a electronic interview. In particular, the $\mathrm{CE}$ analysis asked people to evaluate several alternatives which are described by different levels of attributes, allowing the attributes to be assessed as well as situational changes [6]. To obtain the information about the respondents' preferences over reuse attributes, a specific on line questionnaire was developed (Fig. 4). In particular, the questionnaire comprised four parts. The first asked a series of questions regarding peoples' attitudes towards the particular 
Table 2: Attributes and levels for the evaluation.

\begin{tabular}{ll}
\hline Attributes & Levels \\
\hline Quality of accommodation & Hotel (three stars) \\
& Hotel (two stars) \\
Quality of food & Hostel \\
& Restaurant and Bar \\
& Bar and Fast Food \\
Number of outdoor sports & Fast Food \\
& High \\
Specialized staff following & Medium \\
persons in activities & Low \\
& High \\
Number of indoor sports & Medium \\
& Low \\
Price & Few but large \\
& Medium \\
\hline
\end{tabular}

intervention under analysis; the second part concerned the CE questions; the third section collected opinions about sports activities while the last part was dedicated to the socioeconomic information about respondents (age, gender, municipality of residence, job, income etc.). The completion of the questionnaire was carried out by a group of 150 respondents randomly selected online.
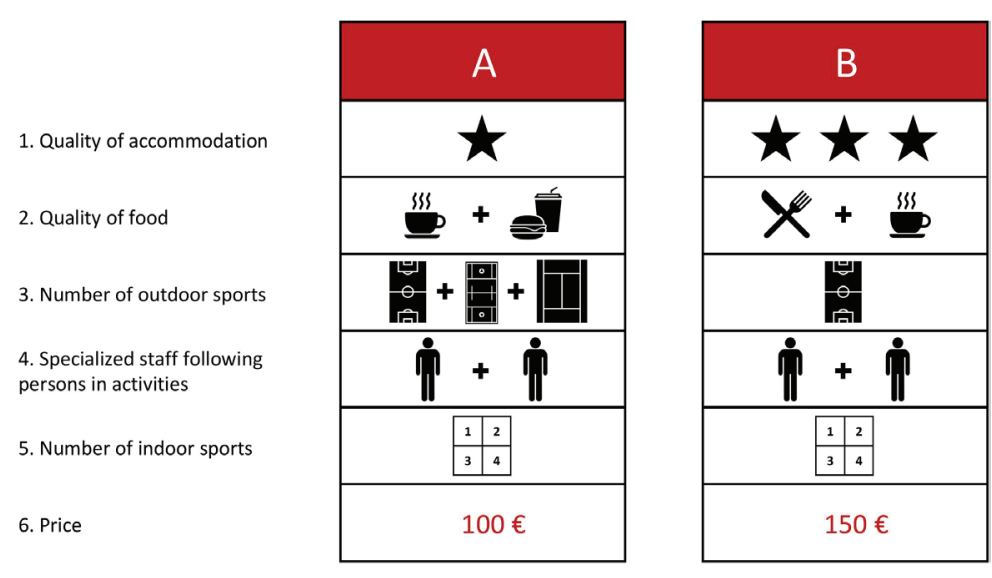

Figure 4: Example of a choice set of the on line questionnaire. 


\subsection{Econometric model and implicit prices}

The answers to the CE questionnaire were analyzed within the Random Utility Model framework [9]. Responses were run through the software SPSS in order to estimate the probability of a given choice being made as a function of its characteristics. Both linear and logit regression were estimated and the results are represented in the first two columns of Table 3.

As it is possible to see, the attributes related to staff, number of outdoor activities and number of indoor sports resulted to be not statistically significant in both the regression models. For this reason these attributes were excluded from the analysis and new results were calculated. The last two columns of Table 3 summarizes the final results of the regression models. As it is possible to see the attributes related to accommodation and food have a positive sign, meaning that they positively affect the choice while the price has a negative sign, affecting in a negative way the probability of choosing one option. From the results of Table 3 it is also possible to affirm that the respondents considered the food as much more important than the accommodation for the selection of the different options.

The analysis of the data collected with the CE survey can be useful also to assess how much individuals value project concepts. For this purpose, the estimation coefficients of the partial model in Table 2 are used to evaluate the degree to which the interviewees do tradeoffs among the attributes. In particular, from the parameters of the model it is possible to calculate the marginal Willingness To Pay (WTP) for each attribute and to estimate the WTP for a specific option. According to [17], the marginal WTP (or implicit price) for each attribute is calculated as minus the ratio between the estimate of the coefficient for the attribute under examination and the monetary coefficient as in:

$$
W T P=-\frac{\beta_{c}}{\beta_{y}}
$$

where $\beta_{c}$ represents the coefficient of any attributes, and $\beta_{y}$ represents the coefficient of cost.

The application of the formula provides the implicit prices. According to the calculations done, the respondents would be willing to pay $22 €$ for increasing of one level the quality of the accommodation (i.e. for passing from a two stars hotel to a three stars hotel). Following

Table 3: Results of regression models.

\begin{tabular}{lllll}
\hline & $\begin{array}{c}\text { Logistic } \\
\text { Linear regression } \\
\text { (complete model) }\end{array}$ & $\begin{array}{c}\text { regression } \\
\text { (complete } \\
\text { model) }\end{array}$ & $\begin{array}{c}\text { Linear regression } \\
\text { (partial model) }\end{array}$ & $\begin{array}{c}\text { Logistic } \\
\text { regression } \\
\text { (partial } \\
\text { model) }\end{array}$ \\
\hline (Constant) & $0.140(1.545)$ & -0.224 & $0,403(10,264)^{* * *}$ & 0.420 \\
Staff & $-0.029(-1.277)$ & -0.077 & $/$ & $/$ \\
Accommodation & $0.072(3.388)^{* * * *}$ & $0.196^{* * *}$ & $0.050(4.333)^{* * * *}$ & $0.217^{* * * *}$ \\
Food & $0.173(8.730)^{* * *}$ & $0.432^{* * *}$ & $0.106(9.687)^{* * *}$ & $0.451^{* * *}$ \\
Outdoor & $0.019(0.925)$ & 0.055 & $/$ & $/$ \\
Indoor & $-0.007(-0.343)$ & -0.023 & $/$ & $/$ \\
Price & $-0,186(-9.802)^{* * *}$ & $-0.010^{* * *}$ & $-0.002(-9.795)^{* * *}$ & $-0.010^{* * *}$ \\
\hline$* * *$ significance level of 0.05 & & &
\end{tabular}


the same reasoning, respondents would be willing to pay $45 €$ for increasing of one level the quality of the food (i.e. for passing from a fast food to a restaurant).

\section{DISCUSSION OF THE RESULTS AND CONCLUSIONS}

The findings of the evaluation allowed to define some guidelines for supporting the design of the reuse project of the new 'Saint Gréé Sports House' (Fig. 5). In particular, the WTP is the data that clearly expresses the individuals' preferences and it permitted to understand on what attributes one should act to improve qualitative and quantitative aspects in the project.

The high WTP on the quality of accommodation and on the quality of food that has been detected from the application of the CE model is reflected in the project by the inclusion of the highest quality standards (threes star hotels and restaurants with bar). Given the large size of the structure, it has been considered as appropriate to ensure a wider choice in the quality of accommodation and, over the three stars hotel, a smaller part destined to hostel. As far as the other three attributes of the evaluation model are considered, as mentioned before, these resulted to be not significant from a statistics point of view. However, from the point of view of the project it has been decided to insert outdoor sports, such as sports fields, mountain bike trails and trekking. The indoor sports, that will be designed and integrated into the structure, will consider few activities but with large spaces. All activities included will be designed and studied to require a minimum number of specialized staff.

The experimentation of CE presented in the paper aimed at supporting the decision-making process about the possibility of restoring the building, stimulating new visions and opportunities. The results of the CE application could be useful for the local Municipality for supporting the development of an action plan for the regeneration of the complex. Indeed, the results could be employed for the preparation of practical guidelines for leading the intervention of private developers potentially interested in the requalification of the building. Moreover, the WTP figures would allow us to estimate the overall benefits provided by specific reuse projects that could be used for the construction of more complete Cost Benefit Analysis model of the overall operation [25]. In particular, by means of this study it would be possible to understand if tangible and intangible benefits of the reuse project outweigh its cost.

This paper represents one attempt of addressing the issues related to integrated assessment and re-use strategies in a very degraded context for formulating innovative sustainable solutions. In this sense, it can be put in evidence that how to reuse abandoned buildings has become an important topic of discussion in sustainable territorial development. Indeed, abandoned buildings represent important cultural, economic and environmental capital as they can provide spaces for new activities reducing land consumption and the use of other natural resources,

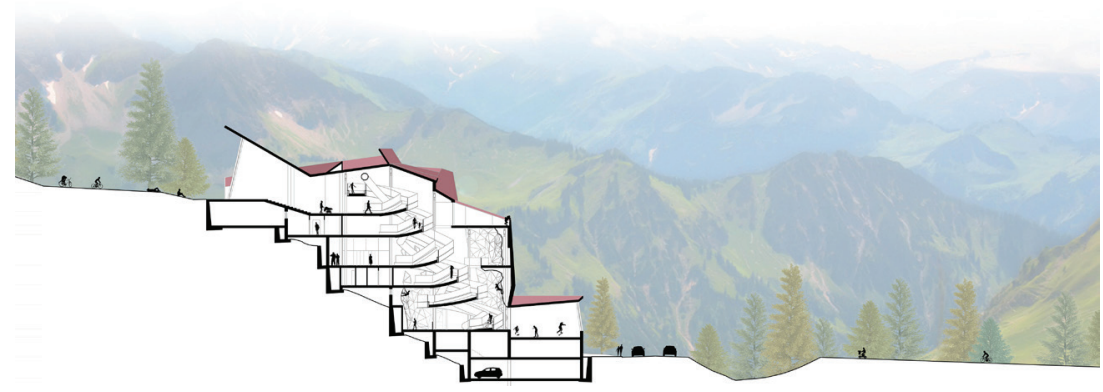

Figure 5: Section of the project of Saint Gréé di Viola. 
offering also social and economic benefits [26]. In this context an emerging approach is related to the so called 'adaptive re-use'. Even if different definitions exist of adaptive re-use, it has been broadly recognized that adaptive re-use involves operations that respect the historic significance of the building adding new and more contemporary layers for increasing the asset value in the future [18], [27]. Following this reasoning, the paradigm of adaptive re-use imposes to respect tangible and intangible aspects of the building under examination and it seems to be a promising strategy in the context of sustainable development seeking for a balance among resources efficiency, cost reduction and valorization of intrinsic values.

The research seems to have an high innovative value because it represents one of the first experimentation of the $\mathrm{CE}$ method for supporting the design process of alternative visions. In fact, according to [19], most of the evaluation applications start with a set of alternatives that is 'given' whereas it is well known that in real world decision situations the set of options is not very often given, but it has to be constructed as the rest of the evaluation model. The use of the $\mathrm{CE}$ proved to be effective in leading the identification of the most relevant features needed for the design and implementation of a successful reuse project for the building under investigation.

Future developments of the study may explore the combined use of CE and Multicriteria Analysis methods [20] for the elicitation of preference information and the evaluation of the alternatives according to a multi-methodological approach [21], [22], [24], [28].

\section{REFERENCES}

[1] Bottero, M. \& Mondini, G., Assessing socio-economic sustainability of urban regeneration programs: An integrated approach. Smart and Sustainable Planning for Cities and Regions, pp. 165-184, 2017. https://doi.org/10.1007/978-3-319-44899-2_10

[2] Tyler, P., Warnock, C., Provins A. \& Lanz, B., Valuing the benefits of urban regeneration. Urban studies, 50(1), pp. 169-190, 2013. https://doi.org/10.1177/0042098012452321

[3] Lancaster, K.J., A new approach to Consumer Theory. The Journal of Political Economy, 74(2), pp. 132-157, 1966. https://doi.org/10.1086/259131

[4] Adamowicz, W., Boxall, P. \& Williams, M., Stated preference approaches for measuring passive use values. Choice experiments and contingent valuation. American Journal of Agricultural Economics, 80(1), pp. 64-75, 1998. https://doi.org/10.2307/3180269

[5] Alvarez-Farizo, B. \& Hanley, N., Using conjoint analysis to quantify public preferences over the environmental impacts of wind farms. An example from Spain. Energy Policy, 30(2), pp. 107-116, 2002. https://doi.org/10.1016/s0301-4215(01)00063-5

[6] Tagliaferro, C., Longo, A. \& Van Eetvelde, V., Landscape economic valuation by integrating landscape ecology into landscape economics. Environmental Science \& Policy, 32, pp. 26-36, 2013. https://doi.org/10.1016/j.envsci.2012.12.001

[7] European Commission, Guide to Cost Benefit Analysis for investment projects, DG Regional and Urban Policy, European Commission: Brussel, 2015.

[8] Louviere, J.J., Flynn, T.N. \& Carson, R.T., Discrete Choice Experiments are not Conjoint Analysis. Journal of Choice Modelling, 3(3), pp. 57-72, 2010. https://doi. org/10.1016/s1755-5345(13)70014-9

[9] McFadden, D., The choice theory approach to market research. Marketing Science, 5(4), pp. 275-279, 1986. https://doi.org/10.1287/mksc.5.4.275

[10] Rosato,. P. \& Rotaris, L., La scarsa diffusione delle coperture verdi in Italia: bassa disponibilità a pagare o poca informazione? Valori e Valutazioni, 13, pp. 57-66, 2014.

[11] Rambonilaza, M., \& Dachary-Bernard, J., Land-use planning and public preferences: What can we learn from choice experiment method. Landscape and Urban Planning, 83(4), pp. 318-326, 2007. https://doi.org/10.1016/j.landurbplan.2007.05.013 
[12] Sayadi, S., Gonzalez-Roa, M.C. \& Calatrava Requena, J., Ranking versus scale rating in conjoint analysis: Evaluating landscapes in mountainous regions in southeastern Spain. Ecological Economics, 55(4), pp. 539-550, 2005. https://doi.org/10.1016/j. ecolecon.2004.12.010

[13] De Rossi. A., Architettura alpina moderna in Piemonte e Valle d'Aosta, Umberto Allemandi \& C.: Torino, 2005.

[14] De Rossi \& A., Dini,. R., Architettura alpina contemporanea, Umberto Priuli \& Verlucca: Scarmagno, 2012.

[15] Manganelli, B., Real Estate Investing. Market Analysis, Valuation Techniques And Risk Assessment, Springer: Berlin, 2015.

[16] Adelman, S., Orthogonal main effects plans for asymmetrical experiments. Technometrics, 4(1), pp. 21-46, 1962. https://doi.org/10.2307/1266170

[17] Haneman W.M., Discrete/Continuous models of consumer demand. Econometrica, 52(3), pp. 541-561, 1984. https://doi.org/10.2307/1913464

[18] Oppio A. \& Bottero M., Conflicting values in designing adaptive reuse for cultural heritage. A case study of social multicriteria evaluation. Lecture Notes in Computer Science, 10406 LNCS, pp. 607-623, 2017. https://doi.org/10.1007/978-3-319-62398-6_43

[19] Misirlisoy, D. \& Gunce, K., Assessment of the adaptive reuse of castles as museums: Case of Cyprus. International Journal of Sustainable Development and Planning, 11(2), pp. 147-159, 2016. https://doi.org/10.2495/sdp-v11-n2-147-159

[20] Tsoukias, A., Aiding to decide and evaluation: challenges for the future. Valori $e$ Valutazioni, 13, pp. 33-36, 2014.

[21] Figueira, J., Greco, S. \& Erghott, M. (eds), Multicriteria Decision Aiding, State of the Art Survey, Springer: Berlin, 2005.

[22] Bottero, M., A multi-methodological approach for assessing sustainability of urban projects. Management of Environmental Quality: An International Journal, 26(1), pp. 138-154, 2015. https://doi.org/10.1108/meq-06-2014-0088

[23] Sala, S., Ciuffo, B. \& Nijkamp, P., A systemic framework for sustainability assessment. Ecological Economics, 119, pp. 314-325, 2015. https://doi.org/10.1016/j. ecolecon.2015.09.015

[24] Bottero M., Dell'Anna, F. \& Nappo, M., Evaluating tangible and intangible aspects of cultural heritage: An application of the promethee method for the reuse project of the Ceva-Ormea railway. Integrated Evaluation for the Management of Contemporary Cities, pp. 285-295, 2018. https://doi.org/10.1007/978-3-319-78271-3_23

[25] Becchio, C., Bottero, M.C., Corgnati, S.P. \& Dell'Anna, F., Decision making for sustainable urban energy planning: an integrated evaluation framework of alternative solutions for a NZED (Net Zero-Energy District) in Turin. Land Use Policy, 78, pp. 803-817, 2018. https://doi.org/10.1016/j.landusepol.2018.06.048

[26] Yung, E.H.K. \& Chan, E.H.W., Implementation challenges to the adaptive reuse of heritage buildings: Towards the goals of sustainable, low carbon cities. Habitat International, 36(3), pp. 352-361, 2012. https://doi.org/10.1016/j.habitatint.2011.11.001

[27] Bottero, M., Bravi, M., Dell' Anna, F. \& Mondini, G., Valuing building energy efficient through Hedonic Prices Method: are spatial effects relevant? Valori e Valutazioni, 21, pp. 27-40, 2018.

[28] Brunetta, G., Salizzoni, E., Bottero, M., Monaco, R. \& Assumma, V., Measuring resilience for territorial enhancement: An experimentation in Trentino. Valori e Valutazioni, 20, pp. 69-78, 2018. 